\title{
Uma revisão integrativa dos diagnósticos de enfermagem mais evidenciados no cateterismo cardíaco
}

\author{
An integrative review of the most evidenced nursing diagnoses in cardiac catheterization \\ Una revisión integradora de los diagnósticos de enfermería más comunes en el \\ cateterismo cardíaco
}

Juliane Lima Alencar ${ }^{1}$, Lucas Vinicius Moraes da Silva ${ }^{2}$, Brena Suelen Gama Macias ${ }^{3}$, Débora Dinnaly de Souza Cravo², Luelma Pereira dos Santos ${ }^{2}$, Jessica da Silva Ferreira ${ }^{4}$, Jaqueline Cristina da Silva Belém ${ }^{4}$, Paulo Henrique Viana da Silva ${ }^{2}$, Ana Lúcia da Silva Ferreira ${ }^{4}$, Daniele Melo Sardinha ${ }^{5 *}$.

\section{RESUMO}

Objetivo: Descrever os diagnósticos de enfermagem em pacientes submetidos ao cateterismo cardíaco mais citados na literatura. Métodos: Estudo exploratório, através de uma Revisão Integrativa da Literatura. Nas bases de dados LILACS e BDENF e PubMed sem limite temporal, incluindo artigos originais, nas línguas inglesa, portuguesa e espanhol. Analisou-se os dados pela leitura e organização dos dados em uma tabela, destacando os diagnósticos mais prevalentes. Resultados: A busca resultou-se em 8 artigos. Os diagnósticos evidentes foram: Dor aguda; Integridade da pele prejudicada ou risco de integridade da pele prejudicada; Ansiedade; Mobilidade física prejudicada; Déficit do autocuidado; Risco de perfusão cardíaca diminuída; Risco de resposta adversa a meio de contraste com iodo; Conhecimento deficiente. Considerações finais: Esses diagnósticos estão associados ao procedimento em si pelas características definidoras e outros de riscos de complicações relacionadas ao cateterismo cardíaco e ao paciente hospitalizado em geral, entretanto sabe-se que existem diversos diagnósticos que não foram discutidos, devido a limitação do estudo.

Palavras-chave: Diagnósticos de enfermagem, Cateterismo cardíaco, Cuidados de enfermagem.

\section{ABSTRACT}

Objective: To describe the nursing diagnoses in patients undergoing cardiac catheterization most often cited in the literature. Methods: Exploratory study, through an Integrative Literature Review. In the LILACS, BDENF and PubMed databases with no time limit, including original articles, in English, Portuguese and Spanish. Data was analyzed by reading and organizing the data in a table, highlighting the most prevalent diagnoses. Results: The search resulted in 8 articles. The most prevalent diagnoses were: Acute pain; Impaired skin integrity or risk of impaired skin integrity; Anxiety; Impaired physical mobility; Self-care deficit; Risk of decreased cardiac perfusion; Risk of adverse response to iodine contrast medium; and Poor knowledge. Final considerations: These diagnoses are associated with the procedure itself by the defining characteristics and others of risks of complications related to cardiac catheterization and the hospitalized patient in general, however it is known that there are several diagnoses that were not discussed due to the limitation of the study.

Key words: Nursing diagnoses, Cardiac catheterization, Nursing care.

\section{RESUMEN}

Objetivo: Desvelar los diagnósticos de enfermedad en pacientes sometidos a cateterismo cardíaco más citados en la literatura. Métodos: Estudio exploratorio, a través de una revisión bibliográfica integradora. En

${ }^{1}$ Secretaria de Saúde Pública do Estado do Pará (SESPA), Belém - PA.

2 Centro Universitário Metropolitano da Amazônia (UNIFAMAZ), Belém - PA.

3 Instituto Evandro Chagas (IEC-PPGEVS), Ananindeua - PA.

${ }^{4}$ Faculdade Integrada da Amazônia (FINAMA), Belém - PA.

5 Universidade do Estado do Pará e Instituto Evandro Chagas (UEPA-IEC-PPGBPA), Belém - PA.

*E-mail: danielle-vianna20@hotmail.com

SUBMETIDO EM: 9/2021 
las bases de datos LILACS, BDENF y PubMed sin límite de tiempo, incluyendo artículos originales, en inglés, portugués y español. Los datos se analizaron leyendo y organizando los datos en una tabla, destacando los diagnósticos más frecuentes. Resultados: La búsqueda dio como resultado 8 artículos. Los diagnósticos más frecuentes fueron: Dolor agudo; Deterioro de la integridad de la piel o riesgo de deterioro de la integridad de la piel; Ansiedad; Deterioro de la movilidad física; Déficit de autocuidado; Riesgo de disminución de la perfusión cardíaca; Riesgo de respuesta adversa al medio de contraste de yodo; y Conocimiento deficiente. Consideraciones finales: Estos diagnósticos están asociados al procedimiento en sí por las características definitorias y otras de riesgos de complicaciones relacionadas con el cateterismo cardíaco y el paciente hospitalizado en general, sin embargo se sabe que hay varios diagnósticos que no fueron discutidos debido a la limitación del estudio.

Palabras clave: Diagnósticos de enfermería, Cateterismo cardíaco, Cuidados de enfermería.

\section{INTRODUÇÃO}

As Doenças Cardiovasculares (DC), são um conjunto de condições crônicas patológicas que afetam o sistema cardiocirculatório como: Infarto Agudo do Miocárdio (IAM), Trombose Venosa Profunda (TVP), Embolia Pulmonar (EP), Insuficiência Cardíaca (IC), Aterosclerose etc., que são influenciadas por fatores de riscos modificáveis e não modificáveis, porém destaca-se os principais fatores envolvidos, a Hipertensão Arterial Sistêmica (HAS) e Diabetes Mellitus (DM). Sendo assim as DC representam $31.8 \%$ da causa de todos os óbitos no Brasil, sendo as mais preocupantes as doenças das artérias coronárias (COSTA GF, et al., 2020; MASSA KHC, et al., 2019).

As artérias coronarianas são responsáveis por irrigar e oxigenar o músculo do miocárdio, sendo primordiais para uma diástole e sístole adequada. Desta maneira necessitam manter um fluxo contínuo a fim de atender a demanda de oxigênio exigida pelo miocárdio. A aterosclerose é a formação de uma placa de colesterol, cálcio, macrófagos e outros elementos do sangue, na parede das artérias, ocasionado a diminuição da luz do vaso, influenciada por fatores genéticos e ambientais como a alimentação, obesidade e sedentarismo. Essa condição associada a outros fatores como a HAS, pode estimular a agregação plaquetária no local da placa de ateroma, uma vez que a HAS irá causar atrito sanguíneo na placa de ateroma, esse processo forma um trombo e obstrui total ou parcial a luz do vaso, causando a angina e IAM, são eventos graves e na sua maioria das vezes podem ser fatais (BRUNORI EHFR, et al., 2014).

As apresentações clínicas típicas de isquemia do miocárdio são: dor precordial com irradiação para braço esquerdo e mandíbula, palidez cutânea, dispneia, confusão mental. Associa-se a dor torácica ao IAM, a dor prolongada entre 20 e 30 minutos, e que se mantêm em repouso, vale ressaltar a investigação de fatores de riscos envolvidos para a conduta adequada. Para o diagnóstico necessita-se a realização do Eletrocardiograma (ECG), que através das alterações das ondas mostrará o local da isquemia. Existem ainda a pesquisa por enzimas cardíacas no sangue, que são marcadores de necrose do miocárdio, porém não são confiáveis no início da isquemia, necessita-se realizar a coleta e análise a cada três horas (REED GW, et al., 2017).

Para o tratamento ou diagnóstico das obstruções coronarianas se realiza o cateterismo cardíaco, que é um procedimento invasivo, realizado em uma sala de hemodinâmica, que nada mais é que a inserção de um cateter na artéria radial ou femoral, com o objetivo de injetar contraste nas coronárias e identificar os pontos de obstrução ou se realizar a desobstrução através da angioplastia, com o auxílio de um balão, que após é implantado uma prótese endovascular (stent), usado para manter o fluxo e a luz do vaso, ainda existem o stent farmacológico e o não farmacológico que é o mais utilizado (MANDA YR e BARADHI KM, 2021).

Nesse contexto, para a realização deste procedimento necessita-se de uma equipe multiprofissional especializada como: Médico, Enfermeiro e Técnico de Enfermagem Hemodinamicistas. Por se tratar de um procedimento de alto grau de complexidade, como também com grandes de chances de complicações graves. Destaca-se a atuação do Enfermeiro nesse procedimento, uma vez que é responsável por assistir o paciente no pré, trans e pós-procedimento (COSTA GRI, et al., 2014).

O serviço do profissional Enfermeiro é sustendo a partir das Teorias de Enfermagem e Sistematização da Assistência de Enfermagem (SAE), o que torna o cuidar baseado em evidência e ciência. A SAE é um conjunto 
de medidas que o Enfermeiro deve organizar, com o objetivo de traçar o plano de cuidados. Dentro da SAE existe o Processo de Enfermagem, que nada mais é que a metodologia do passo a passo, composto por seis etapas: 1 - Histórico de Enfermagem; 2 - Diagnóstico de Enfermagem; 3 - Plano Assistencial; 4 - Plano de Cuidados ou Prescrição de Enfermagem; 5 - Resultados Esperados; 6 - Avaliação (GANDOLFI M, et al., 2016).

Sendo assim inicia-se investigando, através do exame físico e histórico, então identifica-se os problemas de enfermagem embasados nas Teorias de Enfermagem. Na segunda etapa é o momento de traçar os diagnósticos de Enfermagem a partir dos problemas levantados, nessa fase utiliza-se a taxonomia de Diagnósticos de Enfermagem da Nanda International (NANDA-I), que possui diversos diagnósticos reais e de riscos. Para cada diagnóstico necessita-se intervenções específicas, com objetivo de alcançar os resultados esperados (JOHN WSL, 2014).

Desta maneira o Enfermeiro atua a partir de evidências cientificas, através de um processo sistematizado, com o auxílio da Taxonomia NANDA, nesta perspectiva emergiu-se a seguinte questão de pesquisa: Quais os Diagnósticos de Enfermagem em pacientes submetidos ao Cateterismo Cardíaco mais citados na literatura? Sendo o objetivo desta pesquisa descrever os diagnósticos de enfermagem em pacientes submetidos ao Cateterismo Cardíaco mais citados na literatura.

\section{MÉTODOS}

Esta pesquisa trata-se de um estudo exploratório do tipo Revisão Integrativa da Literatura (RIL). Sendo muito relevante esse tipo de revisão, uma vez que permite a análise de integrada, ou seja, a partir da inclusão de uma diversidade de metodologias sobre determinado tema, com o objetivo de sintetizar as evidências já presentes na literatura e subsidiar suporte para a tomada de decisão e melhorar a prática clínica. Na realização da RIL deve-se seguir as seis etapas (MENDES KDS, et al., 2008)

Na primeira etapa ocorreu elaboração da questão de pesquisa, na segunda etapa foi definido os critérios para inclusão e exclusão de estudos/ amostragem ou busca na literatura, na terceira etapa foi estabelecido quais devem ser extraídas dos estudos selecionados/ categorização dos estudos, na quarta etapa é a análise dos estudos incluídos na revisão integrativa, na quinta etapa ocorreu a interpretação dos resultados e na sexta etapa realizado a apresentação da revisão/síntese do conhecimento (MENDES KDS, et al., 2008).

Formulou-se a questão de pesquisa a partir da estratégia PICO (Paciente, Intervenção, Comparação e Outcomes/desfecho), como afirma Santos CMDC, et al. (2007), quais os Diagnósticos de Enfermagem em pacientes submetidos ao Cateterismo Cardíaco mais citados na literatura? Paciente: Submetidos ao cateterismo cardíaco de qualquer idade; Intervenção: diagnósticos de enfermagem evidenciados na literatura; Comparação: Não se aplica; Outcome/Desfecho; descrever os diagnósticos de enfermagem no cateterismo cardíaco.

Para responder à questão deste estudo, elegeu-se a busca nas bases de dados eletrônicas Literatura Latino-Americana e do Caribe em Ciências da Saúde (LILACS) e Base de Dados de Enfermagem (BDENF) e PubMed. Utilizando os seguintes descritores: Diagnósticos de Enfermagem; Cateterismo Cardíaco; Cuidados de Enfermagem. Para a potencialização da busca, usou-se o cruzamento dos descritores com o operado boleano AND.

Foram incluídos nessa pesquisa artigos originais, publicados nos idiomas Português, Inglês e Espanhol, sem limite temporal. Para os critérios de exclusão optou-se por não incluir outros estudos de revisão pois aumenta o risco de viés de resultados, uma vez que dentro da revisão pode estar um dos estudos originais já incluídos em nossa revisão. Foi utilizado o fluxograma prisma para a visualização da busca (LIBERATI A, et al., 2009).

As estratégias de palavras chaves utilizadas foram: Diagnósticos de Enfermagem AND cateterismo cardíaco no LILACS resultando em 6 artigos, sendo apenas 2 que se enquadraram nos critérios de inclusão, já com Cuidados de Enfermagem AND Cateterismo Cardíaco resultou em 40 artigos, duplicando os 2 já incluídos. Já na BDENF, a busca com os descritores Diagnósticos de Enfermagem AND Cateterismo Cardíaco 
resultou em 4 artigos que foram excluídos, já utilizando Cuidados de Enfermagem AND Cateterismo Cardíaco resultou em 24 artigos, em que foram inclusos 3 . No PubMed com as palavras-chaves em inglês resultou em 213 e incluiu-se 3: Sendo assim selecionou-se para a amostra final 8 artigos.

Para a análise dos dados procedeu-se na leitura na íntegra dos artigos selecionados pelos autores, após realizado a discussão para a busca do consenso para a definição da amostra. Para a extração dos dados foi utilizado um formulário, instrumento elaborado pelos pesquisadores composto pelos seguintes itens: número do artigo, título, autores, ano e base de dados, metodologia e diagnósticos de enfermagem mais evidenciados. Desta maneira buscou-se na amostra os diagnósticos de enfermagem mais prevalentes no paciente submetido ao cateterismo cardíaco, para posterior discussão.

\section{RESULTADOS}

A amostra final de artigos selecionados foi 8 , sendo 2 da base de dados LILACS e 3 da BDENF, e 3 do PubMed como descreve a Figura 1.

Figura 1 - Fluxograma PRISMA caracterizando a busca nas bases de dados.

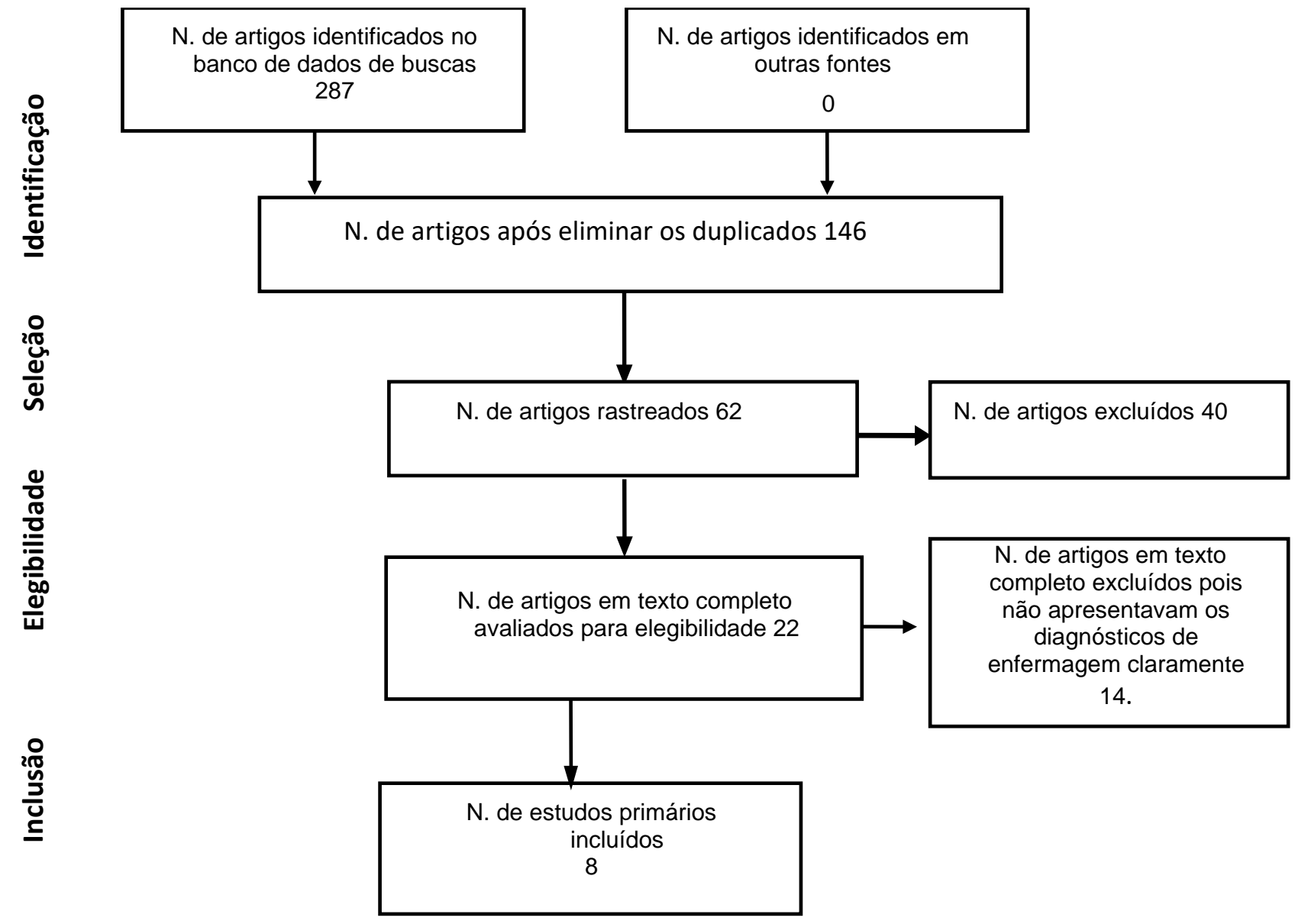

Fonte: Alencar JL, et al., 2021.

Após a seleção da amostra, organizou-se os artigos em uma tabela de acordo om o instrumento utilizado com as seguintes informações: número do artigo, título, autores, ano e base de dados, metodologia e diagnósticos de enfermagem evidenciados (Quadro 1). 
Quadro 1 - Caracterização dos estudos primários incluídos.

\begin{tabular}{|c|c|c|c|}
\hline № Título/Base & Autores & Metodologia & Diagnósticos de Enfermagem \\
\hline $\begin{array}{l}\text { 1- Validação clínica dos diagnósticos } \\
\text { de enfermagem Integridade Tecidual } \\
\text { Prejudicada e Integridade da Pele } \\
\text { Prejudicada em pacientes } \\
\text { submetidos a cateterismo cardíaco- } \\
\text { PUBMED }\end{array}$ & $\begin{array}{l}\text { Paganin A e Rabelo } \\
\text { ER (2013). }\end{array}$ & $\begin{array}{l}\text { Este estudo de validação clínica } \\
\text { usando o modelo de Fehring foi } \\
\text { realizado em um hospital geral } \\
\text { brasileiro entre novembro de } 2009 \text { e } \\
\text { novembro de } 2010 \text {. Este estudo incluiu } \\
250 \text { pacientes que receberam } \\
\text { cateterismo eletivo da artéria femoral. } \\
\text { Cada paciente foi identificado como } \\
\text { tendo duas características de } \\
\text { Integridade Tecidual }\end{array}$ & $\begin{array}{l}\text { A característica definidora de tecido danificado foi } \\
\text { validada para Integridade do tecido prejudicada e as } \\
\text { características definidoras da invasão de estruturas } \\
\text { corporais e o rompimento da superfície da pele foram } \\
\text { validadas para Integridade da pele prejudicada. } \\
\text { Integridade da pele prejudicada }\end{array}$ \\
\hline $\begin{array}{l}2 \text { - Diagnósticos de enfermagem em } \\
\text { pacientes submetidos a cateterismo } \\
\text { cardíaco em uma unidade de } \\
\text { cardiologia-BDENF }\end{array}$ & $\begin{array}{l}\text { Aquino EM, et al. } \\
(2014) \text {. }\end{array}$ & $\begin{array}{l}\text { Estudo exploratório, descritivo, } \\
\text { realizado com sete enfermeiros de } \\
\text { uma unidade de cardiologia de um } \\
\text { hospital universitário de Curitiba/PR. }\end{array}$ & $\begin{array}{l}\text { Mobilidade no leito prejudicada; Risco de perfusão renal } \\
\text { ineficaz; Risco de resposta adversa a meio de } \\
\text { contraste com iodo; Risco de perfusão tissular periférica } \\
\text { ineficaz; Risco de infecção; Risco de sangramento; Risco } \\
\text { de integridade da pele prejudicada; Dor aguda. }\end{array}$ \\
\hline $\begin{array}{l}\text { 3- Um estudo de validação clínica de } \\
\text { mobilidade física prejudicada de } \\
\text { pacientes submetidos a cateterismo } \\
\text { cardíaco-PUBMED }\end{array}$ & $\begin{array}{l}\text { Paganin A e Rabelo } \\
\text { ER (2012). }\end{array}$ & $\begin{array}{l}\text { Estudo transversal, validação clínica } \\
\text { baseada no modelo de Fehring das } \\
\text { características definidoras do } \\
\text { diagnóstico de enfermagem } \\
\text { mobilidade física prejudicada em uma } \\
\text { amostra de } 250 \text { pacientes. }\end{array}$ & $\begin{array}{l}\text { Foram validadas: amplitude de movimento limitada, } \\
\text { capacidade limitada de realizar habilidades motoras } \\
\text { grossas e dificuldade de girar. } \\
\text { Mobilidade física prejudicada. }\end{array}$ \\
\hline $\begin{array}{l}\text { 4- Enfermagem em laboratório de } \\
\text { hemodinâmica: diagnóstico e } \\
\text { intervenção fundamentados na Teoria } \\
\text { da Adaptação de Roy-BDENF }\end{array}$ & $\begin{array}{l}\text { Oliveira MF e Silva } \\
\text { LDF (2010). }\end{array}$ & $\begin{array}{l}\text { Estudo do tipo descritivo e transversal, } \\
\text { quantitativo, realizado em um hospital } \\
\text { público, de Fortaleza-CE, de julho a } \\
\text { setembro de } 2009 \text {, em uma amostra de } \\
233 \text { pacientes. }\end{array}$ & $\begin{array}{l}\text { Risco de infecção; risco de sangramento; comunicação } \\
\text { verbal prejudicada; risco de intolerância a atividade; dor } \\
\text { aguda; risco de perfusão tissular cardíaca diminuída; } \\
\text { déficit no autocuidado para banho; ansiedade; dentição } \\
\text { prejudicada e conforto prejudicado; Integridade da pele } \\
\text { Prejudicada. }\end{array}$ \\
\hline
\end{tabular}




\begin{tabular}{|c|c|c|c|}
\hline № Título/Base & Autores & Metodologia & Diagnósticos de Enfermagem \\
\hline $\begin{array}{l}\text { 5- Intervenções e resultados de } \\
\text { enfermagem para o diagnóstico de } \\
\text { integridade tissular prejudicada em } \\
\text { pacientes após cateterismo cardíaco: } \\
\text { pesquisa-PUBMED }\end{array}$ & $\begin{array}{l}\text { Pezzi MV, et al. } \\
(2016) \text {. }\end{array}$ & $\begin{array}{lcr}\text { Pesquisa } & \text { com } & \text { e-questionários } \\
\text { enviados } & \text { para } & \text { enfermeiras } \\
\text { especialistas } & \text { em duas rodadas. }\end{array}$ & $\begin{array}{l}\text { Apenas um resultado de enfermagem foi aprovado: } \\
\text { integridade do tecido - pele e mucosa e cinco intervenções } \\
\text { relacionadas, a saber, controle de pressão, administração } \\
\text { de medicamentos tópicos, cuidados com o local da } \\
\text { incisão, cuidados com lesões e controle de infecções. } \\
\text { Integridade da pele prejudicada }\end{array}$ \\
\hline $\begin{array}{l}\text { 6- Assistência de enfermagem a } \\
\text { idosos que realizam cateterismo } \\
\text { cardíaco: uma proposta a partir do } \\
\text { modelo de adaptação de Calista Roy- } \\
\text { LILACS }\end{array}$ & $\begin{array}{l}\text { Freitas MC e } \\
\text { Oliveira MF (2006). }\end{array}$ & $\begin{array}{l}\text { Desenvolveu-se em hospital de pronto- } \\
\text { atendimento em cardiologia de } \\
\text { Fortaleza-CE, de janeiro a julho de } \\
2005 \text { Utilizou-se entrevista } \\
\text { semiestruturada, com } 18 \text { idosos de } \\
\text { ambos os sexos, no pré-cateterismo. }\end{array}$ & $\begin{array}{l}\text { Alteração na manutenção da saúde; ansiedade; medo; } \\
\text { alteração no processo familiar }\end{array}$ \\
\hline $\begin{array}{l}7 \text { - Diagnósticos de Enfermagem em } \\
\text { pacientes pós-cateterismo cardíaco - } \\
\text { contribuição de Orem-LILACS }\end{array}$ & $\begin{array}{l}\text { Lima LR, et al. } \\
(2006) .\end{array}$ & $\begin{array}{l}\text { Estudo de múltiplos casos, transversal, } \\
30 \text { clientes, no período pós- } \\
\text { cateterismo cardíaco. Através do } \\
\text { NANDA. O método de coleta de dados } \\
\text { foi o Processo de Enfermagem } \\
\text { fundamentado na Teoria Déficit do } \\
\text { Autocuidado. }\end{array}$ & $\begin{array}{l}\text { Integridade tissular prejudicada; Risco para infecção; Dor } \\
\text { aguda (região inguinal); Mobilidade física prejudicada, } \\
\text { Déficit do autocuidado para higiene íntima; Risco para } \\
\text { lesão orgânica renal. }\end{array}$ \\
\hline $\begin{array}{l}8 \text { - Cuidados de enfermagem no } \\
\text { cateterismo cardíaco e angioplastia } \\
\text { coronariana: desenvolvimento de um } \\
\text { instrumento-BDENF }\end{array}$ & $\begin{array}{l}\text { Régis AP, et al. } \\
(2017) .\end{array}$ & $\begin{array}{l}\text { Pesquisa quanti-qualitativo. Com cinco } \\
\text { Enfermeiros, as pesquisadoras } \\
\text { elaboraram um checklist, baseando-se } \\
\text { nas etapas do processo de } \\
\text { enfermagem. }\end{array}$ & $\begin{array}{l}\text { Ansiedade/Medo; Risco de Sangramento; Risco de } \\
\text { Resposta Alérgica; Dor Aguda; Conhecimento Deficiente; } \\
\text { Risco de Integridade da Pele Prejudicada; Risco de } \\
\text { Perfusão Tissular Periférica Ineficaz. }\end{array}$ \\
\hline
\end{tabular}

Fonte: Alencar JL, et al., 2021. 
Foi possível através do instrumento para a coleta de dados, organizar e descrever as principais características da amostra. Desta maneira dos 8 artigos selecionados, todos abordam sobre os diagnósticos de enfermagem no cateterismo cardíaco, em que foi realizado baseado na Taxonomia NANDA. Porém o artigo 6 focou apenas nos diagnósticos psicossociais, embasados no modelo de adaptação de calista roy, mostrando que o artigo 4 também se baseou nesse modelo, porém abordou os diagnósticos não somente nos aspectos psicossociais. Quanto ao ano de publicação destas pesquisas: 1-2013, 2-2014, 3-2012, 4-2010, 5-2016. 6-2006, 7-2006 e 8-2017.

Após a análise dos diagnósticos na tabela destacou-se os mais evidentes: Dor aguda; Integridade da pele prejudicada; Risco de integridade da pele prejudicada; Ansiedade; Mobilidade física prejudicada; Déficit do autocuidado; Risco de perfusão cardíaca diminuída; Risco de resposta adversa a meio de contraste com iodo; Conhecimento deficiente.

\section{DISCUSSÃO}

Esta revisão integrou metodologias de pesquisas que relataram os diagnósticos de enfermagem no cateterismo cardíaco, e mostrou que todos se basearam na referência internacional o NANDA, o que fortalece a qualidade dos estudos incluídos para a síntese desta pesquisa.

Após a descrição dos diagnósticos mais prevalentes nesta revisão mostrou-se que o mais evidente foi a Dor Aguda. A dor é uma condição sensitiva e emocional desagradável, relacionada a um agravo real ou potencial, em algum tecido. Desta maneira o seu manejo adequado é fundamental, com o objetivo de melhorar a qualidade vida do indivíduo em vários aspectos, sendo comum após um procedimento invasivo que causa danos a algum tecidos (FILHO GSF, et al., 2012).

O diagnóstico da dor aguda se identifica a partir do relato do paciente, uma vez que é uma informação subjetivo, entretanto procedimentos invasivos como cateterismo cardíaco já se espera a presença da dor. Desta maneia cabe ao enfermeiro avaliar o nível da dor, administrar corretamente os analgésicos prescritos, manter dose extras se necessário, proporcionar ambiente confortável e posições que minimizem a dor, dentro das limitações recomendadas para a recuperação. O alívio da dor oferece qualidade de vida e sucesso na recuperação do paciente (O'KEEFE-MCCARTHY S, et al., 2015).

Um estudo utilizou a massagem terapêutica e houve uma redução altamente significativa nos escores de dor após as sessões de massagem terapêutica, e houve uma diferença altamente significativa entre antes e depois da intervenção das sessões de mensagem em relação a todos os componentes e pontuação média total do perfil de humor. Concluíram que integrar a massagem terapêutica à intervenção de enfermagem pode melhorar o humor e o estado de habilidade e diminuir a ansiedade, as depressões, a confusão e a dor dos pacientes após o cateterismo cardíaco (HASSAN HE, et al., 2019).

Outro diagnóstico prevalente foi o Integridade da pele prejudica. Que por sua vez tem como características definidoras qualquer dano no tecido cutâneo, relacionado ou não a procedimentos invasivos (JOHN WSL, 2014). O procedimento é partir de um dano mecânico (introdução do cateter na artéria femoral durante o cateterismo cardíaco) caracterizada por lesão na região inguinal, que necessita cuidados, com o intuito de impedir colonização e infecção. Medidas assépticas são recomendadas, como também a utilização das precauções padrão (CHEN T, et al., 2021).

Este estudo também evidenciou que o cateterismo cardíaco ocasiona ansiedade nos pacientes. Corroborando com uma pesquisa com 100 pacientes (30 mulheres e 70 homens), com média de idade de 61 \pm 9 anos na sala de espera de um laboratório de hemodinâmica (pré-cateterismo). Entre os pacientes, $30 \%$ tinham níveis de ansiedade altos a muito altos. Eles estavam preocupados com complicações e dores. Apenas $52 \%$ dos pacientes relataram ter recebido informações pré-intervenção e $65 \%$ disseram que assinaram consentimento sem lê-lo. $67 \%$ dos pacientes ficaram satisfeitos com o atendimento da equipe (BEJAR MA, et al., 2021).

Desta maneira o Enfermeiro deve na consulta pré cateterismo, esclarecer todas as dúvidas e medos do paciente, como também explicando todo o passo a passo do procedimento, com o objetivo de o paciente 
saber tudo que irá acontecer e minimizando as chances de entrar em pânico ou medo, pois sabe-se que a ansiedade altera alguns parâmetros como, frequência cardíaca, respiração, e ainda causa estresse, sendo esses fatores não favoráveis para a recuperação adequada (OLIVEIRA AA, et al., 2018).

Destacou-se também o diagnóstico de Mobilidade Física Prejudicada. Indo ao encontro com o resultado de Sartori AA, et al. (2018), que investigou os diagnósticos de enfermagem em pacientes no setor de hemodinâmica, com 100 pacientes, e que a Mobilidade Física Prejudicada foi evidenciada em 50\% dos pacientes, sendo ainda relacionado a punção arterial, pois por se tratar de um dano ao tecido, ainda requer repouso, pois trata-se de uma lesão em uma artéria, apresentando alto risco de hemorragias e complicações que podem levar a óbito.

As intervenções do Enfermeiro para a Mobilidade Física Prejudica após cateterismo cardíaco, deve ser orientar o paciente para repouso absoluto no leito, por no mínimo 12 horas sem mobilizar o membro que foi realizado a punção e monitorar os pulsos. Sabe-se que o cateterismo cardíaco apresenta chances de complicações potenciais como: risco de sangramento, hematoma no local da punção, pseudoaneurisma, traumatismo decorrente da cateterização, formação de coágulo, vasoespasmo e até IAM (AQUINO EM, et al., 2014).

Mostrou-se ainda que o cateterismo cardíaco é um procedimento que compromete o autocuidado do paciente, pois evidenciou-se o diagnóstico de enfermagem Déficit do Autocuidado. Destacado na pesquisa de Costa GF, et al. (2020), em que avaliou 6 artigos em uma revisão integrativa sobre os diagnósticos de enfermagem na angioplastia transluminal e mostrou que o Déficit do Autocuidado foi um dos diagnósticos mais ocorrentes, em que se encontrou na maioria dos estudos incluídos em sua pesquisa.

Ficando evidente que o cateterismo cardíaco compromete a autonomia do autocuidado dos pacientes, uma vez que exige repouso absoluto nas primeiras horas, e ainda necessitando que o paciente não faça nenhum esforço até a recuperação completa. Nesse contexto o Enfermeiro deve promover os cuidados de higiene básico para esses pacientes, sabe-se que a higiene e primordial para a saúde e recuperação adequada (REED GW, et al., 2017; RÈGIS AP, et al., 2017).

O Risco de Perfusão Cardíaca Diminuída também foi prevalente nesta revisão. Sendo este diagnóstico caracterizado pelo risco de redução na circulação cardíaca (coronárias). Este diagnóstico anteriormente era titulado como perfusão tissular ineficaz. Sendo esta mudança influenciada a partir dos indicadores de adaptação do componente da oxigenação e esteve associada à hipóxia cardíaca secundária e à interrupção do fluxo sanguíneo (SARTORI AA, et al., 2018).

Para os cuidados realizados pela equipe de enfermagem nesse diagnóstico são realizar monitoração contínua do eletrocardiograma, observando atentamente as alterações nos níveis pressóricos (no início aumenta, após a instalação do choque cardiogênico reduz) desconforto respiratório, pele fria, sudorese, aumento da frequência cardíaca inicial e redução após a fadiga cardíaca, atentar para saturação de oxigênio, realizar o balanço hídrico (esperado redução do débito). Outras recomendações envolvem posição supina e membros inferiores elevados para aumentar a pré-carga, manter acesso venoso calibroso, administrar drogas da terapêutica e deixa o carro de parada pronto para uma intercorrência (OLIVEIRA MF e SILVA LDF, 2010).

O Risco de resposta adversa a meio de contraste com iodo, foi um Diagnóstico de Enfermagem também destacado. Sendo assim têm-se como característica definidora, qualquer reação relacionada ao uso do iodo no procedimento, respostas alérgicas ou nefrotóxicas, merecendo atenção até sete dias depois da sua utilização (SARDINHA DM, et al., 2020). O enfermeiro orientar quanto a pausa de fármacos rotineiros do paciente (especificamente antidiabéticos orais), histórico de alergias. As reações adversas podem ser leves, moderadas ou graves, sendo assim utiliza-se alguns medicamentos preventivos, que incluem a combinação de corticosteróides e anti-histamínicos, capazes de minimizar essas reações (AQUINO EM, et al., 2014).

Mostrou também que o diagnóstico Conhecimento Deficiente foi relatado. Esse diagnóstico tem como característica definidora a presença da falta de conhecimento do paciente relacionado ao cateterismo cardíaco, pois o "não saber" irá ocasionar tensão, medo e ansiedade, nesse sentido o enfermeiro deve orientar o paciente sobre o porquê de o paciente necessitar do cateterismo cardíaco, como será realizado, e os 
cuidados após os procedimentos, e minimizar os fatores de riscos cardiovasculares que levaram o paciente até o procedimento. Com o objetivo de tranquilizar o paciente e sensibiliza-lo para o autocuidado em relação ao tratamento de sua condição cardíaca e minimização dos fatores de riscos cardiovasculares, a fim de oferecer qualidade de vida para esse indivíduo (OLIVEIRA AA, et al., 2018).

Nesta perspectiva a SAE tem como objetivo oferecer uma assistência de qualidade, repercutindo na segurança do paciente. Através dos Diagnósticos de Enfermagem e possível realizar esse cuidado baseado em evidência científica, sistematizando o serviço. No cateterismo cardíaco o paciente estará exposto a diversos problemas de enfermagem, sendo primordial o Enfermeiro assisti-lo de forma integral e holística, pois os diagnósticos não se prendem somente ao procedimento em si, como também, envolve outros fatores que poderão ou não interferir no sucesso do procedimento ou recuperação, pois a SAE deve ser individualiza, garantindo intervir nos problemas reais apresentados por cada indivíduo (SILVA EGC, et al., 2011).

\section{CONSIDERAÇÕES FINAIS}

Foi possível evidenciar os Diagnósticos de Enfermagem mais prevalentes no paciente submetido ao Cateterismo Cardíaco: Dor aguda; Integridade da pele prejudicada ou risco de integridade da pele prejudicada; Ansiedade; Mobilidade física prejudicada; Déficit do autocuidado; Risco de perfusão cardíaca diminuída; Risco de resposta adversa a meio de contraste com iodo; Conhecimento deficiente. Esses diagnósticos estão associados ao procedimento em si pelas características definidoras e outros de riscos de complicações relacionadas ao cateterismo cardíaco e ao paciente hospitalizado em geral. Entretanto sabe-se que existem diversos diagnósticos que não foram discutidos, devido a limitação do estudo, que também refletiu na inclusão dos artigos atuais na revisão, mostrando a necessidade de estudo originais e atuais sobre o tema.

\section{REFERÊNCIAS}

1. AQUINO EM, et al. Diagnósticos de enfermagem em pacientes submetidos a cateterismo cardíaco em uma unidade de cardiologia. Rev enferm UFPE online, 2014; 11(8): 3929-37.

2. BEJAR MA, et al. Anxiety in the cardiac catheter laboratory: Importance of the education. Archives of Cardiovascular Diseases Supplements, 2021; 13(2): 195.

3. BRUNORI EHFR, et al. Association of cardiovascular risk factors with the different presentations of acute coronary syndrome. Revista Latino-Americana de Enfermagem, 2014; 22(4) :538-546.

4. CHEN T. Application progress of imaging examination and cardiac catheterization in diagnosis, treatment and longterm management of cardiovascular sequelae of Kawasaki disease: interpretation of the Japanese Circulation Society/Japanese Society for Cardiovascular Surgery 2020 Guideline on Diagnosis and Management of Cardiovascular Sequelae in Kawasaki Disease. Chinese Journal of Applied Clinical Pediatrics, 2021; (24): 561-565.

5. COSTA GRI, et al. Atuação do enfermeiro no serviço de hemodinâmica: uma revisão integrativa. Revista Interdisciplinar, 2014; 7(3): 157-164.

6. COSTA GF, et al. Nursing Care for Patients Submitted to Coronary Transluminal Angioplasty: An Integrative Review. Cardiology and Angiology: An International Journal, 2020; 9(1): 18-26.

7. FILHO GSF, et al. Dor aguda: julgamento clínico de enfermagem no pós-operatório de cirurgia cardíaca. Revista Mineira de Enfermagem, 2012; 16(3):400-409.

8. FREITAS MC, OLIVEIRA MF. Assistência de enfermagem a idosos que realizam cateterismo cardíaco: uma proposta a partir do modelo de adaptação de Calista Roy. Revista Brasileira de Enfermagem, 2006; 59(5): 642-646.

9. GANDOLFI M, et al. Sistematização Da Assistência De Enfermagem: Da Teoria Ao Cuidado Integral. Rev Enferm UFPE online, 2016; 10(4): 3694-3703.

10. HASSAN HE, et al. Effect of Massage Therapy on the Mood and Pain of Post Cardiac Catheterization Patients. American Journal of Nursing Research, 2019; 7(3): 392-399.

11. JOHN WSL. Diagnosticos de enfermagem NANDA 2015-2017. 10ª ed. 2014.

12. LIBERATI A, et al. The PRISMA Statement for Reporting Systematic Reviews and Meta-Analyses of Studies That Evaluate Health Care Interventions: Explanation and Elaboration. PLoS Medicine, 2009; 6(7): 1000-100.

13. LIMA LR, et al. Diagnósticos de Enfermagem em pacientes pós-cateterismo cardíaco: contribuição de Orem. Revista Brasileira de Enfermagem, 2006; 59(3): 285-290.

14. MANDA YR, BARADHI KM. Cardiac Catheterization Risks and Complications. StatPearls, 2021.

15. MASSA KHC, et al. Análise da prevalência de doenças cardiovasculares e fatores associados em idosos, $2000-2010$. Ciência \& Saúde Coletiva, 2019; 24(1): 105-114. 
16. MENDES KDS, et al. Revisão integrativa: método de pesquisa para a incorporação de evidências na saúde e na enfermagem. Texto \& Contexto Enferm, 2008; 17(4): 758-764.

17. OLIVEIRA AA, et al. O cateterismo cardíaco e a enfermagem: a importância dos diagnósticos de enfermagem para uma assistência de qualidade. Revista Recien - Revista Científica de Enfermagem, 2018; 8(23): 21.

18. OLIVEIRA MF, SILVA LDF. Enfermagem em laboratório de hemodinâmica: diagnóstico e intervenção fundamentados na Teoria da Adaptação de Roy. Revista Eletrônica de Enfermagem, 2010; 12(4): 678-68.

19. O'KEEFE-MCCARTHY S, et al. Dor e ansiedade em pacientes rurais com síndrome coronariana aguda que aguardam diagnóstico de cateterismo cardíaco. The Journal of Cardiovascular Nursing, 2015; 30(6): 546-557.

20. PAGANIN A, RABELO ER. A Clinical Validation Study of Impaired Physical Mobility of Patients Submitted to Cardiac Catheterization. International Journal of Nursing Knowledge, 2012; 23(3): 159-162..

21. PAGANIN A, RABELO ER. Clinical validation of the nursing diagnoses of Impaired Tissue Integrity and Impaired Skin Integrity in patients subjected to cardiac catheterization. Journal of Advanced Nursing, 2013; 69(6): 1338-1345.

22. PEZZI MV, et al. Nursing Interventions and Outcomes for the Diagnosis of Impaired Tissue Integrity in Patients After Cardiac Catheterization: Survey. International Journal of Nursing Knowledge, 2016; 27(4): 215-219.

23. REED GW, et al. Acute myocardial infarction. The Lancet, 2017; 389(10065): 197-210.

24. RÉGIS AP, et al. Cuidados de enfermagem no cateterisno cardíaco e angioplastia coronariana: desenvolvimento de um instrumento. Revista Recien - Revista Científica de Enfermagem, 2017; 7(21): 3.

25. SARDINHA DM. et al. Risk Factors for Acute Renal Failure after Cardiac Catheterization Most Cited in the Literature: An Integrative Review. International journal of environmental research and public health, 2020; 17(10).

26. SARTORI AA, et al. Diagnósticos de enfermagem no setor de hemodinâmica: uma perspectiva adaptativa. Rev Esc Enferm USP, 2018; 52(55): 1-8.

27. SILVA EGC, et al. O conhecimento do enfermeiro sobre a Sistematização da Assistência de Enfermagem: da teoria à prática. Revista da Escola de Enfermagem da USP, 2011; 45(6): 1380-1386. 